\title{
Analytical Model Nonequilibrium Inhomogeneous Plasma of the Heliosphere
}

This paper was downloaded from TechRxiv (https://www.techrxiv.org).

\section{LICENSE}

CC BY 4.0

SUBMISSION DATE / POSTED DATE

$05-12-2021 / 13-12-2021$

\section{CITATION}

Vysikaylo, Philipp (2021): Analytical Model Nonequilibrium Inhomogeneous Plasma of the Heliosphere. TechRxiv. Preprint. https://doi.org/10.36227/techrxiv.17126342.v1

DOI

10.36227/techrxiv.17126342.v1 


\section{Analytical Model Nonequilibrium Inhomogeneous Plasma of the Heliosphere}

\section{Philipp I. Vysikaylo}

\begin{abstract}
We prove that a nonequilibrium inhomogeneous giant gas discharge is realized in the heliosphere with huge values of the parameter $E / N$, which determines the temperature of electrons. This quasi-stationary discharge determines the main parameters of the weak solar wind (SW) in the heliosphere. In connection with the development of space technologies and the human spacewalk, the problem of the nature of the $\mathrm{SW}$ is acute. The study of the interference of gravitational and electrical potentials at the Earth's surface began with the work of Hilbert 1600. Such polarization effects - the interference of Coulomb and gravitational forces - have not been studied well enough even in the heliosphere. Our article is devoted to this problem. Pannekoek-Rosseland-Eddington model do not take into account the important role of highly energetic running (away from the Sun) electrons and, accordingly, the duality of electron fluxes. According to an alternative model formulated by we, highly energetic (escaping from the Sun) electrons leave the Sun and the heliosphere, and weakly energetic ones, unable to leave the Coulomb potential well (hole) - the positively charged Sun and the heliosphere, return to the Sun. The weak difference between the opposite currents of highly energetic (escaping from the Sun) electrons and weakly energetic (returning to the Sun) electrons is compensated by the current of positive ions and protons from the Sun - SW. These dynamic processes maintain a quasi-constant effective dynamic charge of the Sun and the entire heliosphere. At the same time, quasi-neutrality in the Sun and heliosphere is well performed up to $10^{-36}$. According to experiments and analytical calculations based on our model: 1) the plasma in the corona is nonequilibrium; 2) the maximum electron temperature is $T_{e} \sim 1-2$ million degrees; 3) $T_{e}$ grows from $1000 \mathrm{~km}$ away from the Sun and 4) the role of highly energetic electrons escaping from the plasma leads to a significant increase in the effective: solar charge and electric fields in the heliosphere in relation to the Pannekoek-Rosseland-Eddington model. This is due to the absence of a compensation layer that screens the effective charge of the Sun. It is not formed at all due to the escape of highly energetic electrons (as in a conventional gas discharge) in the entire heliosphere with high temperatures exceeding the temperature of the Sun's surface. Thus, the process of escape of highly energetic electrons forms the internal EMF of the entire heliosphere. Interference of gravitational and Coulomb potentials in the entire heliosphere is considered, it is being manifested in generation of two opposite flows of particles: 1) that are neutral or with a small charge (to the Sun), and 2) in the form of high-energy electrons (escaping from the positively charged Sun) and a solar wind (from the Sun). Calculated values of the registered ion parameters in the solar wind were compared with experimental observations. Reasons for generating the ring current in inhomogeneous heliosphere and inapplicability of the Debye theory in describing processes in the solar wind (plasma with current) are considerede.
\end{abstract}

Index Terms - solar wind, electroneutrality, gravitational interactions, Coulomb interactions, proton, alpha particle.

Manuscript received 05122021

P.I. Vysikaylo is with the Plasma Chemistry Laboratory, Moscow Radiotechnical Institute RAS, 117519 Moscow, Russia (e-mail: filvys@ yandex.ru).

\section{INTRODUCTION}

The study of the interference of gravitational and electrical potentials at the Earth's surface began with the work of Hilbert [1]. In Hilbert's experiments, the negligible charge of the petals of the same name, constantly received from the high- temperature plasma of the candle, led to their constant levitation in the gravitational field of the Earth due to Coulomb forces. Such polarization effects - the interference of Coulomb and gravitational forces - have not been studied well enough even in the heliosphere. Our article is devoted to the problem of studying the main parameters of the SW in the inner heliosphere and their coordination with the variety of observed heavy positively charged ions in the solar wind.

The idea of the need to take into account the processes of plasma polarization in stars and star spheres was first formulated by Pannekoek in 1922 [2] and Rosseland in 1924 [3]. They took into account only the difference in gravity in the atmosphere of the Sun due to the difference in the masses of electrons and positive ions. Due to this, heavier ions are held by gravity more efficiently than electrons around any star, including the Sun. According to this model, an electric field is created at sizes larger than the Debye radius, compensating for the tendency to particle separation in any gravitating plasmoid. The elementary theory of this charge separation for the static heliosphere was created quite a long time ago and it is constantly being modified until now. The corresponding electric field that returns electrons to the star is called the Pannekoek-Rosseland field. In 1926, Eddington analytically estimated the effective positive charge of the Sun $\approx 300 \mathrm{C}$ using this method [4]. Having received negligible electric fields on the surface of the Sun itself, Eddington said, "the effect is absurdly weak" on the surface of the Sun. And most of the astrophysicists believed that the role of the effective charge of the Sun and galaxies, and, consequently, eddy currents on the charged structures of the Cosmos, is negligible everywhere and is important only on the size of the Debye radius. But, already the Sun's charge of $300 \mathrm{C}$ corresponds to the appearance of reflecting Coulomb mirrors not only for protons, but also for alpha particles. This was first noted in [5].

Polarization models that take into account the global separation of charges due to polarization plasma (in the heliosphere) caused by the difference in the gravitational masses of protons and electrons (main SW components) have been studied in a large number of works with and without taking into account the magnetic field (Lemaire \& Scherer 1971; Lemaire \& Scherer 1973; Pierrard \& Lemaire 1996, 1998; Maksimovic et al. 1997; Scudder 1992; Pierrard, Issautier, Meyer-Vernet and Lemaire 2000; Scudder 2019; Halekas, Whittlesey, Larson, McGinnis, Maksimovic, Berthomier, Kasper, Case, Korreck, Stevens, Klein, Bale, MacDowall, Pulupa, Malaspina, Goetz, and Harvey 2021; and etc.) [6]. However, until now it has not been possible to explain all the parameters and effects from the surface of the Sun to the Earth, observed in the plasma of the heliosphere. Therefore, we have proposed a new SW model operating in the entire inner heliosphere from the surface of the Sun to the Earth's orbit [7].

Even the presence of global electric field of PannekoekRosseland should lead to global electric currents in the heliosphere. We prove that global currents flow in the plasma of the heliosphere from the positively charged Sun to the negatively charged Earth, and all the laws of conventional gas- 
discharge plasma discovered and studied by Stoletov, Townsend, Pashen, etc. work in the entire heliosphere.

\section{GENERAL AND DIFFERENCES IN OUR MODEL AND PANNEKOEK-ROSSELAND-EDDINGTON'S MODEL}

As noted earlier, Pannekoek 1922 put forward the idea that the difference in masses of protons and electrons should lead to charge separation and the generation of polarizing electric fields in the heliosphere. In order to maintain quasi-neutrality in the presence of mass-dependent gravitational forces, electric fields must exist (Pannekoek 1922). Given the large thermal velocity of the light electrons, a considerable electric potential drop should exist between the solar corona and 1 AU (Lemaire \& Scherer 1971). A class of "exospheric" models (Lemaire \& Scherer 1973) posits that this electric field accelerates the solar wind ions from the corona, with the pervasive non-thermal nature of the electron distribution increasing the efficiency of this acceleration (Pierrard \& Lemaire 1996; Maksimovic et al. 1997; Scudder 1992). This electric field may self-consistently generate the nonthermal features of the electron distribution through a runaway process (Scudder 2019). The main relations in these works are the following equations [6]:

$$
\begin{aligned}
& \sum Z_{\mathrm{i}} n_{\mathrm{i}}=n_{\mathrm{e}} \\
& \sum Z_{\mathrm{i}} n_{\mathrm{i}} v_{\mathrm{i}}=n_{\mathrm{e}} v_{\mathrm{e}}
\end{aligned}
$$

The electric field that returns electrons to the star according to the model (1)-(2) is called the Pannekoek-Rosseland field. The movements of charged particles according to Model 1-2 are shown in Fig. $1 a$.
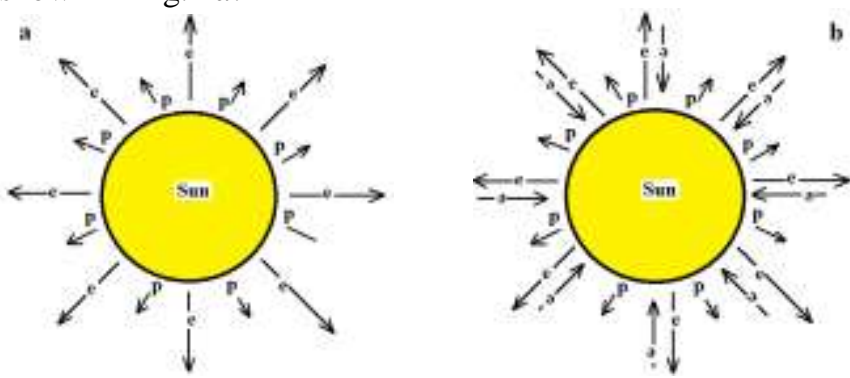

Fig.1. Schemes of the movement of electrons - e and positively charged ions - in the heliosphere: a) - according to model (1-2) and b) - according to our model (3-4) ( $p$ - positively charged ions with $Z / M$ $\geq 0.107)$.

Model (1) - (2) does not take into account the duality of the electron current (Fig.1a). Our model takes into account this duality of the electron current (Fig.1b). High-energy electrons can even leave the potential well - the positively charged Sun and the heliosphere, while the less energetic ones are slowed down by the electric field in the heliosphere and return back to the positively charged Sun. In our model, quasi-constant fluxes of electrons to the positively charged Sun are practically equal to the flux of high-energy electrons from the Sun and differ by the value of the current of positive ions from the Sun. In contrast to model (1) - (2), we have:

$$
n_{\mathrm{e} 2} v_{\mathrm{e} 2}=n_{\mathrm{e} 1} v_{\mathrm{e} 1}+\sum Z_{\mathrm{i}} n_{\mathrm{i}} v_{\mathrm{i}}
$$

Instead of the identical equality (1), we believe that there is a weak violation of electroneutrality on the Sun and throughout the inner heliosphere. The presence of free electrons in the Sun and heliosphere and their high mobility lead to the escape of some high-energy electrons from the Sun. The higher the temperature of the electrons, the greater the part of the electrons capable of leaving the heliosphere (Fig. 2). This leads to an increase in the effective charge of the Sun and a corresponding increase the velocities $v_{1}, v_{2}, v_{\mathrm{i}}$ and penetration of the electric field of the effective charge of the Sun into the entire heliosphere, where the electron temperature is significantly higher than on the Sun's surface and reaches 1-2 million degrees in the Sun's corona. In Fig. 1, we took into account that the main current of positive ions is associated with protons and alpha particles. (Positive ion fluxes are also divided by the interference of gravitational and Coulomb potentials into fluxes to the Sun at $\mathrm{Z} / \mathrm{M}<0.107$ and fluxes from the Sun at $\mathrm{Z} / \mathrm{M} \geq$ 0.107, see Fig. 3 and section III). At this temperature, the formation of a compensatory layer of the positive charge of the Sun does not occur, and the electric field of this quasi-constant charge of the Sun penetrates into the entire heliosphere. This field heats electrons (this leads to the growth of $v_{1}$ and $v_{2}$ in (3)) and accelerates positively charged ions, protons and alpha particles from the Sun (at $Z / M \geq 0.107$ ). The plasma wind of positive ions (mainly protons) carries electrons with it. This is how the solar wind from the Sun is formed according to our model.

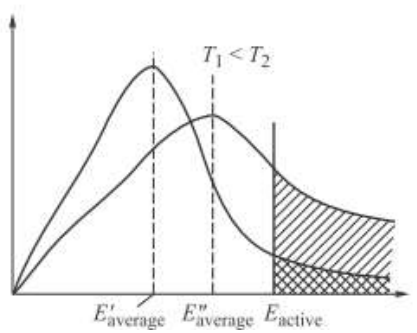

Fig.2. Electron energy distribution functions depending on temperature

Consider the condition of quasi-neutrality and penetration of electric fields in a plasma with a global current. In the scientific and educational literature, various and contradictory definitions of plasma quasi-neutrality are given. The concept of "quasi" is almost. So, quasi-permanent - almost permanent. Without the prefix "quasi", the plasma becomes electrically neutral or, according to the Poisson equation, the density of the space charge in the plasma must be zero everywhere. Therefore, it is necessary to apply equation (1) carefully when modeling a discharge in the heliosphere. In the general case, a reasonable definition of the quasi-neutrality of a simple plasma is:

$$
\alpha_{\mathrm{i}}=\left(n_{\mathrm{i}}-n_{\mathrm{e}}\right) /\left(n_{\mathrm{i}}+N\right) \ll 1
$$

Where $n_{\mathrm{e}}$ is the electron concentration; $n_{\mathrm{i}}$ is the concentration of positive ions; $N$ is the concentration of neutral particles (atoms, molecules, etc.) The question remains open: how many times is $\alpha_{\mathrm{i}}$ less than 1 in the entire heliosphere and in the Sun?

\section{CALCULATIONS OF THE EFFECTIVE POSITIVELY SUN CHARGE AND POSITIVE ION PARAMETERS IN THE SOLAR WIND}

In the heliosphere, two forces act on positively charged ions: the force of gravity and the force of the electric field. If we assume that the electric field is determined by the uncompensated charge of the Sun, then we can estimate the 
effective charge of the Sun from the ionic composition of positive ions in SW [7]. Experimenters in [8,9] found that the following positive ions are observed in SW: $\mathrm{C} 4+, \mathrm{O} 5+, \mathrm{Ne} 8+$, $\mathrm{Mg} 6+, \mathrm{Si}^{+}, \mathrm{Fe} 6+, \ldots, \mathrm{Fe} 13+\ldots(\mathrm{Z} / \mathrm{M}=\ldots=0.33 ; 0.31 ; 0.39$; $0.24 ; 0.21 ; 0.107 \ldots)$. In SW there are heavy positive ions ionized 4 or more times. The absence of positive $\mathrm{Fe} 5+$ ions in SW (Fig.3) gives us the opportunity to assume that for them the forces of Coulomb repulsion from the positively charged Sun are less than the force of gravitational attraction. Hence the effective positive charge of the Sun is $\mathrm{Q}_{s} \approx 1,400 \mathrm{C}$ [7]. With this effective charge of the Sun, all positive ions with $\mathrm{Z} / \mathrm{M} \geq$ 0.107 fly from it, since for them the gravitational forces are less than the Coulomb forces [7].

If there are no negative charges in the heliosphere up to the Earth that could implement Debye's shielding of the effective charge of the Sun, then knowledge of the parameters of the Sun allows us to determine: 1) the electric field strength on the solar surface at $E\left(R_{\mathrm{S}}\right) \approx 2.7 \cdot 10^{-5} \mathrm{~V} / \mathrm{m}$ и 2$)$ the electric field profile in the entire heliosphere $E(R)=K Q_{\mathrm{S}} / R^{2}$ (Fig.4).

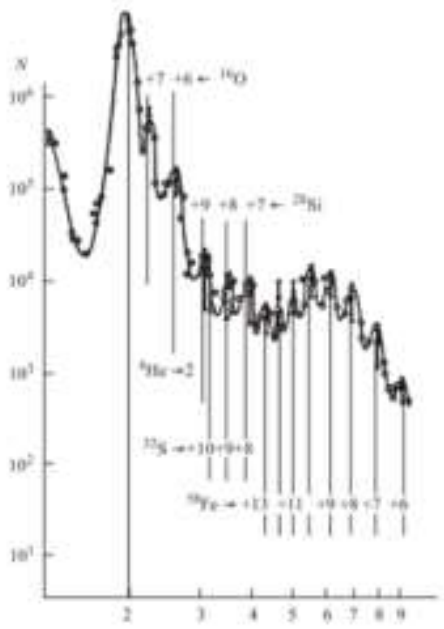

Fig. 3. Typical ion spectrum in the solar wind, measured on the Prognos (Forecast)-7 satellite in 1978-1979. On the horizontal axis the ratio of the mass of particles $m$ to its charge in $10 \mathrm{~s}$. The numbers with the sign "+" denote the charge number of the ion. The proton peak with $Z / M=1$ is not shown, since it exceeds the peak of $\alpha$-particles by more than an order of magnitude [9].

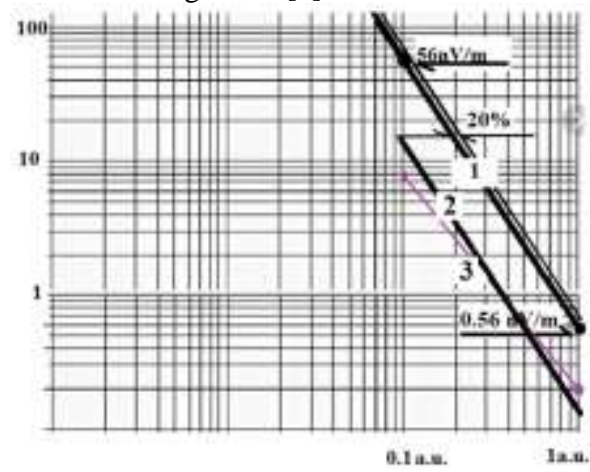

Fig. 4. Calculations of electric fields in the heliosphere: 1- according to the spectrum of positive ions in the solar wind [7] ( $\mathrm{Qs}=1,420 \mathrm{C}$; error less than 20\%); 2 - according to the Eddington's calculations [4]; 3 - according to the calculations of the electric field based on experimental observations of the electron distribution function using the Parker solar probe [6] (Qs $=200 \div 500 \mathrm{C}$, the dependency 3 is built by J.S. Halekas).
The results obtained by us when taking into account the types of ions in SW in [7] are compared with the results obtained by Eddington [4] (electric field strength and the solar charge differ by 4.2 times) and J. S. Halekas et al. [6] in Fig. 4, dependency 3.

\section{THE ELECTRON TEMPERATURE PROFILE IN THE HELIOSPHERE}

According to Paschen's law, the energy of a charged particle is determined not only by the $E(R)$ - electric field (EF) strength, but also by the path length, where a charged particle gains energy in the EF without collisions, i.e., by the $N(R)$ particle number density or by the $E / N$ parameter. This law is used here to describe "mysterious" phenomena in heliosphere inhomogeneous in the $N(R)$ [10-13]. If an increase in $E$ leads to an increase in the temperature of electrons, then a decrease in the density of the number of particles - $N$ (participating in the scattering of electron energy) leads to the same effect.

Table

Dependencies of profiles $N_{1}[11], N_{2}[10,12,13]$ and $E / N$ [Td] (set by the solar charge) depending on the altitude above the Sun surface

\begin{tabular}{|c|c|c|c|c|c|}
\hline$R, \mathrm{~km}=R, R_{\mathrm{s}}$ & $\begin{array}{c}N_{1}[11], \\
\mathrm{cm}^{-3}\end{array}$ & $\begin{array}{c}\mathrm{N}_{2}[10] \\
\mathrm{cm}^{3}\end{array}$ & $\begin{array}{c}E / N_{i} \\
\text { Td }\end{array}$ & $E N=T d$ & $T_{n}, \mathrm{~K} / \mathrm{h}$ \\
\hline 0 & & $1.5 \cdot 10^{17}$ & & & 6430 \\
\hline 91 & & $70^{2 !}$ & & & 5410 \\
\hline 200 & & & & & $4170[12]$ \\
\hline 320 & & $1,3-10^{n}$ & & & 4560 \\
\hline 520 & & $2.4 \cdot 10^{23}$ & & & 4190 \\
\hline 560 & & $1.6 \cdot 10^{21}$ & & & $1 / 80$ \\
\hline $0 / 1$ & $3.9 \cdot 10^{15}$ & & $6.7 \cdot 10^{-6}$ & & \\
\hline 1,000 & $3.1 \cdot 10^{13}$ & & $8.4 \cdot 10^{3}$ & & \\
\hline 1,004 & & $2,5 \cdot 10^{21}$ & & $1.06 \cdot 10^{1}$ & 5750 \\
\hline 1,580 & & $4.10^{11}$ & & $0.066^{\circ}$ & 7550 \\
\hline 1,900 & & $1.3 \cdot 10^{\prime \prime}$ & & $0.2^{\circ}$ & $375^{*}$ \\
\hline 200 & $6.3 \cdot 10^{12}$ & & $4.2 \cdot 10^{3}$ & & \\
\hline 2,0 & & $10^{n}$ & & $0.26^{\circ}$ & $460^{\circ}$ \\
\hline $2,1 / 1,003$ & & $1 \theta^{\rho}$ & & $26.6^{\circ}$ & $11.553^{\circ}$ \\
\hline 3,000 & $2.0 \cdot 10^{12}$ & & $1.3 \cdot 10^{-3}$ & & $30 \% 0.7$ \\
\hline $3,50001.0005$ & & $6-10^{2}$ & & $44^{\circ}$ & $16,697^{\circ}$ \\
\hline 4,000 & $7.9 \cdot 10^{11}$ & & $3.310^{2}$ & & \\
\hline 6,000 & $2.51 \cdot 10^{7}$ & & 10.6 & & $6,164^{*}: 0,7$ \\
\hline 11.01 & & 3.2 .10 & & $83^{\circ}$ & $26,035 * / 0.7$ \\
\hline 7,000 & & $5 \cdot 10^{\circ}[12]$ & & & $25,000^{*}$ \\
\hline 8,000 & $10^{4}$ & & 26 & & $11,5 * 0.7$ \\
\hline 10,000 & $6.3-10^{3}$ & & 40.8 & & $15,84 * 10.7$ \\
\hline 14,000 & & $5 \cdot 10^{3}[12]$ & & & $3\left(\times 0.000^{\circ}\right.$ \\
\hline 15,000 & $2.0-10^{5}$ & & 126 & & $34,872 * 0.7$ \\
\hline 11,03 & & $2+100^{3}$ & & $133^{\circ}$ & \\
\hline 17.06 & & $1.6 \cdot 70^{5}$ & & $166^{\circ}$ & \\
\hline 70,000 & $7.9-10^{7}$ & & 279 & & $60,833 * 0.7$ \\
\hline 70,000 & & & & & $2 \cdot 10^{6}[13]$ \\
\hline 11.1 & & $10^{x}$ & & $233^{\circ}$ & \\
\hline 11.2 & & $67 \cdot 10^{\circ}$ & & & \\
\hline 11.2 & & $4 \cdot 10^{7}[12]$ & & & $1.510^{67}[12]$ \\
\hline 280,000 & $1.2 \cdot 10^{7}$ & & 1,079 & & $156,794 * 00.7$ \\
\hline 11.3 & & $2 \cdot 10^{\circ}$ & & & \\
\hline 420,000 & $5.0-10^{\circ}$ & & 2,074 & & $247,732 * / 0.7$ \\
\hline$/ I, 4$ & & 8.106 & & & \\
\hline 700,000 & $1.6 \cdot 10^{6}$ & & 4,204 & & $406,236 * 0.7$ \\
\hline$/ 2$ & & $3.3 \cdot 10^{2}$ & & & \\
\hline 12 & & $100[13]$ & & & \\
\hline$/ 2.5$ & & 10 & & & \\
\hline 1400,000 & $4.0 \cdot 10^{5}$ & & 7,421 & & $0.9-10^{*} \times 0.7$ \\
\hline 13 & & $2.8 \cdot 60^{5}$ & & & \\
\hline$/ 4$ & & $1.5 \cdot 10^{3}$ & & & \\
\hline 15 & & $10^{3}[13]$ & & & \\
\hline 2800,000 & $6.3 \cdot 10^{2}$ & & 16,851 & & $10^{\circ} / 0.7$ \\
\hline 35 & & $5.0 \cdot 10^{6}$ & & & \\
\hline $6.6[12]$ & $3.1 \cdot 10^{2}$ & 97 & & & \\
\hline 6200,000 & $10^{4}$ & & 26,45 & & $1.5 \cdot 10^{\circ} \times 0.7$ \\
\hline
\end{tabular}




\begin{tabular}{|c|c|c|c|c|c|}
\hline 100 & & $10^{\prime}$ & & 25,7 & \\
\hline 110 & & $10^{4}[12]$ & & & \\
\hline 111 & & $10^{2}[13]$ & & & \\
\hline 13000,000 & $2.5 \cdot 10^{3}$ & & 26,450 & & $1.5 \cdot 10^{6^{*}} / 0,7$ \\
\hline 120 & & $1.6 \cdot 10^{\prime}$ & & $41,260^{*}$ & \\
\hline 150 & & $1.6 \cdot \mathrm{In}^{2}$ & & $66,540^{\circ}$ & $2, \dot{8} \cdot 1 \theta^{2} / 0.7$ \\
\hline 150 & & $10^{2}[12]$ & & & \\
\hline 44000,65 & $10^{3}$ & & 6,290 & & $0.55 \cdot 10^{5}: 0.7$ \\
\hline 1100 & & $3.1 \cdot 10^{7}$ & & $85,830^{\circ}$ & \\
\hline 150000,000 & $6.3 \cdot 10^{2}$ & & 909 & & $0.18-10^{8} / 0.7$ \\
\hline$/ 215$ & & 5 & & $114,500)^{\circ}$ & $4+1 \theta^{6}+0.7$ \\
\hline 215 & & $2.5[12]$ & & & $6.4 \cdot 10^{6} / 0.7$ \\
\hline 1214 & & & & & $10^{5}[13]$ \\
\hline
\end{tabular}

As can be seen from the table, the data in various literary sources agree well in the central region of the inner heliosphere. A significant difference between the data in [10] and the data in [11] is observed in the near corona of the Sun $(R \sim 1000 \mathrm{~km})$ and in the region 1 a.u. Here the difference reaches up to $10^{2}$.

We construct the electron temperature profile $-T_{\mathrm{e}}(R)$ in the entire heliosphere based on the Nernst - Townsend (EinsteinSmolukhovsky) relation [7]:

$T_{\mathrm{e}}=\mathrm{e} D_{\mathrm{e}} / \mu_{\mathrm{e}} \sim(E / N)^{\zeta}$

Calculations (5) for the data in the table and Fig. 5 were carried out under the assumption that the concentrations of electrons are equal to the concentration of hydrogen molecules in the entire heliosphere. If we take into account that in the region of the surface and the corona of the Sun, the neutral gas is atomic hydrogen or even proton gas, then the temperature profiles will rise even more sharply in Fig. 5 near the Sun.

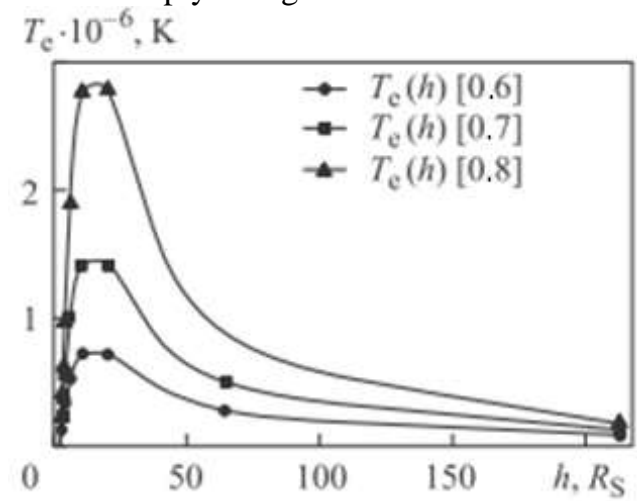

Fig. 5. Electron temperature dependence on distance to the Sun at the [ל] parameter values with the Sun charge of 1,400 C [7].

\section{CONCLUSIONS}

Heavy ions are formed in the corona, and their state does not change when moving in SW, due to the absence of collisions in the heliosphere. Consequently, SW ions carry information about conditions in the solar corona. SW observations are of practical importance. According to the types of observed positive ions in SW, according to [7], we can determine the magnitude of the effective quasi-constant positive charge of the Sun. Knowing the profile of the electric field $E(R)$, we can calculate the energy of charged particles born in the sun's corona and compare it with the energy of particles observed experimentally in the Earth's orbit (in the solar wind). These comparisons are in good agreement with an effective charge of the Sun of 1400 C [7]. At the Sun charge determined in [4] (Fig. 4, line 2), the proton energy is 4.5 times less than that observed in a slow SW, and when determined in [6] (Fig. 4, line 3), it is 7 times less than that observed in a slow
SW [15]. Verification of mathematical models of the heliosphere should be carried out in a comprehensive manner, taking into account all the facts observed by us experimentally, taking into account the types of positive ions observed in SW (Fig. 3). In all models (see ref. in [6]), the types of positive ions were not taken into account in determining the effective Sun charge and the incorrect model 1-2 (fig.1a) was used, not 3-4 (fig. $1 b$ ) as in our case.

For the first time as a result of analytical studies of ring current in the heliosphere global electric circuit, according to model 3-4, we have calculated the parameters of the heliosphere and verified by experimental observations:

1) effective positive Sun charge is $1,400 \mathrm{C}$;

2) with such a positive charge, the Sun is able to reflect all positively charged particles with the ratio (of the charge number $-\mathrm{Z}$ to the mass number $-\mathrm{M}) \mathrm{Z} / \mathrm{M} \geq 0.107$. These are such positively charged particles as: protons, alpha particles and multiply ionized ions of heavy atoms $(\mathrm{C} 4+, \mathrm{O} 5+, \mathrm{Ne} 8+$, $\mathrm{Mg} 6+, \mathrm{Si6}+, \mathrm{Fe} 6+, \ldots, \mathrm{Fe} 13+$ generated in the solar corona), observed in the SW $[8,9,15]$;

3 ) protons and alpha particles velocities arising at distances from the Sun of $(10 \div 30) \cdot R_{\mathrm{s}}$. They are accelerating in EF of the positively charged Sun up to $400 \mathrm{~km} / \mathrm{s}$ and more in the Earth area;

4) non-compensation coefficient of the Sun positive charge $\alpha_{i}$ $=7.5 \cdot 10^{-36}$, i.e., for $10^{36}$ compensated nucleons there are only 7.5 electrons that left the Sun far beyond the problem scope (for example, the Solar system);

5) $E / N$ parameter profile for heliosphere, which determines the breakdown conditions and the electron temperature, the $E(R)$ EF strength profile was calculated according to the Coulomb law, and the profile of nucleon density in heliosphere was taken from [10-13];

6) the $T_{\mathrm{e}}$ profile in the entire heliosphere (from the Sun to the Earth) was calculated using the Nernst - Townsend (Einstein - Smoluchowski) relation for the $\mathrm{E} / \mathrm{N}$ profile according to (5) [7]. The $T_{\mathrm{e}}$ profile (Fig. 5) with $\zeta=0.7$ is in good agreement with experimental observations, see table.

The electric field of the positively charged Sun penetrates into the entire heliosphere, as in the entire heliosphere, as well as in the Sun itself, constant fluxes of electrons escaping from the Sun are formed. In laboratory plasma, an external EMF is usually used (electric batteries, power plants, etc.). For a laboratory gas discharge, the particle number density usually changes slightly, and in the heliosphere up to $10^{17}$ times from the surface of the Sun to the Earth's orbit. For the heliosphere, where the density of gas particles, $N$, falls faster than the electric field strength from the distance to the Sun, a solar charge of 1,400 C leads to the generation of breakdown values of $E / N$ already at altitudes of the order of $1000 \mathrm{~km}$. At altitudes of 10-30 solar radii, a non-equilibrium gas-discharge plasma is formed with huge values of the $E / N$ parameter and, consequently, electron temperatures up to 1 - 2 million degrees (see table, Fig. 5). At such temperatures, global eddy currents are formed in the entire heliosphere (Fig. 1b), caused by the escape of high-energy electrons from the Sun [7]. As proved in our works, this is associated with a sharp drop in the number of particles $N$, on which the relaxation of the electron energy occurs. 
According to our ideas, a giant discharge is realized in the heliosphere between the positively charged Sun and negatively charged planets (for example, the Earth). In our model, the Sun is an analogue of the anode, and the negatively charged Earth is the cathode. In the heliosphere, the negatively charged Earth (as a cathode) is surrounded by the positively charged ionosphere of the Earth - an analogue of a positively charged cathode spot, on which there is a significant drop in the external potential - cathode drop. The large charge of the Earth $(500 \mathrm{kC}$ [14]) in comparison with the effective charge of the Sun $(1,400$ $C$ [7]) can be explained by a significant voltage drop across the positively charged ionosphere. The positively charged heliosphere itself is analogous to the positive column of plasma in a laboratory discharge. For this reason, the heliosphere is positively charged and does not have a compensation layer for the positive charge of the Sun at distances from the Sun at the size of the Debye radius. In a laboratory gas discharge, the EMF is usually outside the discharge gap, and in the heliosphere, the EMF of such a giant discharge is due to the escape of highenergy electrons from the entire heliosphere and from the Sun and the processes of electron-electron collisions, leading to the transfer of energy to the tail of the electron energy distribution function (in the region of energies characteristic of runaway electrons [7]). The intrusion of high-energy electrons escaping from the Sun into the planets leads to a negative charge of the planets (in particular, the Earth up to $500 \mathrm{kC}$ [14]). If we take into account the presence of such a global gas inhomogeneous nonequilibrium discharge, one can explain many phenomena observed in the heliosphere, which are similar to those in a laboratory discharge.

The role of high energy electrons escaping from plasma structures in the formation and propagation of anode-directed plasma structures has already been well studied in the scientific literature [16]. In this case, the electrons are accelerated by the electric field and fly out of the plasma structure against the direction of the electric field $-E$, without collisions leading to the loss of their energy. The escaping high energy electrons provide preionization and transition of the non-activated gas medium into plasma. In our work, it was proven for the first time that escaping free high energy electrons from the plasma structure - the heliosphere (or electrons in the tail of the electron distribution function) lead to the formation of standing shock waves of an electric field reduced to density $N$ (see Table and Fig. 5). This happens due to a sharp decrease in the concentration of particles $-N$ (inhomogeneous due to the presence of gravitational forces) on which the energy of escaping electrons is relaxed. This phenomenon observed in the heliosphere proves the asymmetry of the influence of the electric field $-E$ and density $-N$ on the plasma parameters in the heliosphere [7], as in a laboratory gas discharge (see Townsend's and Stoletov's works).

We have two methods for the experimental determination of the local electric field (Fig. 3 curves 1 [7] and 3 [6]). Based on the results of the work [7], we can explain all the phenomena observed in the heliosphere. Based on the results of the work [6], we cannot explain the presence of positive ions $\mathrm{Fe}+6,7,8$ in the solar wind, the temperature of electrons in 1-2 million degrees in the heliosphere. This indicates that the method [6] requires significant modification.

\section{REFERENCES}

1. https://www.peoples.ru/science/physics/william_gilbert/

2. Pannekoek, A., Ionization in stellar atmospheres, Bull. Astron. Inst. Neth., 1, 107, 1922.

3. Rosseland S., "Electrical state of a Star," Monthly Notices of the Royal Astronomical Society, Vol. 84, No. 308, 1924, pp. 720-728.

http://articles.adsabs.harvard.edu/pdf/1924MNRAS..84..720R

4. Eddington A.S. The internal constitution of the stars. Cambridge Univ. Press, 1926.

5. Vysikaylo, P.I. Surf. Engin. Appl.Electrochem. (2012) 48, №3, p. 212. https://doi.org/10.3103/S106837551203012X

6. J. S. Halekas, P. Whittlesey, D. E. Larson, D. McGinnis, M. Maksimovic, M. Berthomier, J. C. Kasper, A. W. Case, K. E. Korreck, M. L. Stevens, K. G. Klein, S. D. Bale, R. J. MacDowall, M. P. Pulupa, D. M. Malaspina, K. Goetz, and P. R. Harvey. Electrons in the Young Solar Wind: First Results from the Parker Solar Probe https://arxiv.org/abs/1912.02216v1

7. Vysikaylo P.I., Korotkova M.A. Determination of the Sun's charge by the parameters of heavy ions in the solar wind. J.

Phys., Conf. Ser., 2018, vol. 1009, art. 012020. https://doi.org/10.1088/1742-6596/1009/1/012020

8. http://www.srl.caltech.edu/ACE/ASC/level2/

9. Ermolaev Yu.I. Eksperimental'noe isuchenie krupnomasshtabnoy struktury solnechnogo vetra. Dis. d-ra fiz.-mat. nauk [Experimental study on large-scale structure of solar wind. Doc. Sc. Eng. Diss.]. Moscow, Space Research Institute, 2002 (in Russ.).

10. Allen K.U. Astrofizicheskie velichiny (Spravochnik). Izdatel'stvo: Mir. 1977. 279 s.

https://www.studmed.ru/allen-ku-astrofizicheskie-velichinyspravochnik_5d9e8d105eb.html

11. Martynov D.Ya. Kurs obshchey astrofiziki. M.: Nauka,1971 -616s 12. Monin A.S. Global hydrodynamics of the sun. // Sov. Phys. Usp. 23 594-619 (1980). https://ufn.ru/en/articles/1980/9/e/

13. Fizicheskaya entsiklopediya / Gl. red. A. M. Prokhorov. - M.: Bol'shaya Rossiyskaya entsiklopediya, 1994. - T. 4. Poyntinga Robertsona - Strimery. - S. 579-580. -704 s. ISBN 852700878 https://ru.wikipedia.org/wiki/Физическая_энциклопедия

14. Smirnov B.M. Electrical cycle in the Earth's atmosphere // Phys. Usp. 57 1041-1062 (2014). https://ufn.ru/en/articles/2014/11/a/

15. Plazmennaya geliogeofizika $v 2$ t. / Pod red. L.M. Zelenogo, I.S. Veselovskogo. - M.: FIZMATLIT, 2008 - 672 i 560 s.

16. Babich L.P. High-energy phenomena in electric discharges in dense gases: theory, experiment and natural phenomena. ISSN 12345678. Futurepast Inc., Arlington, Virginia, USA, 2003. https://www.studmed.ru/babich-lp-high-energy-phenomena-inelectric-discharges-in-dense-gases-theory-experiment-and-naturalphenomena_10ebc03d518.html

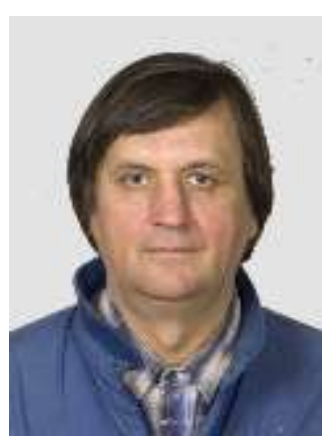

Philipp I. Vysikaylo, is the lead researcher of Moscow Radiotechnical Institute, RAS. He received MS degree in experimental nuclear Physics from the Moscow Institute for Physics and Technology, Moscow, Russia, the Ph.D. in plasma physics and chemistry from Kurchatov Institute of Nuclear Energy, and Dr. of Sciences degree from M.V. Lomonosov Moscow State University, Moscow Russia. He was Expert of the Ministry of the Russian Federation for Atomic Energy (State Institution State Scientific and Technical Center of Expertise of Projects and Technologies - SI SSTCEPT). He is the expert of the Russian Foundation for Basic Researches. He has 46 year experience in plasma physics, namely, in the physics of elementary processes, gas discharges, electron-beam plasmas, plasma chemistry and lightning. 